Check for updates

Cite this: Soft Matter, 2021, 17, 3976

DOI: 10.1039/d1sm90055b

rsc.li/soft-matter-journal

\section{Correction: Characterizing surface wetting and interfacial properties using enhanced sampling (SWIPES) $\dagger$}

\author{
Hao Jiang, (D) Suruchi Fialoke, Zachariah Vicars (D) and Amish J. Patel (D)*
}

Correction for 'Characterizing surface wetting and interfacial properties using enhanced sampling (SWIPES)' by Hao Jiang et al., Soft Matter, 2019, 15, 860-869, DOI: 10.1039/C8SM02317D.

The authors regret their use of the average fluid center of mass, $\left\langle x_{\mathrm{COM}}\right\rangle_{\kappa, N^{*}}$, as a proxy for the vapor-liquid interface location, $H$. Although both $\left\langle x_{\mathrm{COM}}\right\rangle_{\kappa, N^{*}} \approx H$ and $h_{\mathrm{COM}} \equiv \mathrm{d}\left\langle x_{\mathrm{COM}}\right\rangle_{\kappa, N^{*}} / \mathrm{d} N^{*} \approx h$ are excellent approximations for thin surfaces, these assumptions can lead to systematic error in $h$ for thicker surfaces. In particular, in the ESI for this correction notice, $\dagger$ we use simple geometric arguments to show:

$$
\frac{h_{\mathrm{COM}}}{h} \approx 1-\alpha \lambda
$$

where $\alpha$ is the fraction of the simulation box taken up by the surface along the $z$-axis, and $\lambda$ is the fraction of water molecules that are outside the observation volume, $v$. Thus, when either the surfaces are thin relative to the liquid slab $(\alpha \rightarrow 0)$, or most of the water molecules in the system are in $v(\lambda \rightarrow 0), h_{\mathrm{COM}} \rightarrow h$.

An alternative, more robust approach for obtaining $h$ is to approximate the location of the vapor-liquid interface, $H$, using the halfdensity isosurface, $x_{\text {int }}^{\kappa, N^{*}}(z)$, for each biased simulation. This isosurface is implicitly defined by $\langle\rho(x, z)\rangle_{\kappa, N^{*}}=\frac{1}{2}\left(\rho_{\mathrm{L}, \mathrm{b}}+\rho_{\mathrm{V}, \mathrm{b}}\right) \approx \frac{1}{2} \rho_{\mathrm{L}, \mathrm{b}}$, where $\rho_{\mathrm{L}, \mathrm{b}}$ and $\rho_{\mathrm{V}, \mathrm{b}}$ are the bulk liquid and vapor densities, respectively. In practice, we obtain $x_{\text {int }}^{\kappa, N^{*}}\left(z_{i}\right)$ at each value $z_{i}$ by fitting $\left\langle\rho\left(x, z_{i}\right)\right\rangle_{\kappa, N^{*}}$ to the sigmoidal function: $\frac{1}{2} \rho_{\mathrm{L}, \mathrm{fit}}\left[1-\tanh \left(\left[x-x_{\text {int }}^{\kappa, N^{*}}\left(z_{i}\right)\right] / d_{\mathrm{fit}}\right)\right]$, where $\rho_{\mathrm{L}, \mathrm{fit}}, d_{\mathrm{fit}}$, and $x_{\text {int }}^{\kappa, N^{*}}\left(z_{i}\right)$ are fit parameters. We then average $x_{\text {int }}^{\kappa, N^{*}}(z)$ over the $z$-axis to obtain $\bar{x}_{\text {int }}^{\kappa, N^{*}}$, and compute $h_{\text {int }}$ as the slope of $\bar{x}_{\text {int }}^{\kappa, N^{*}} v s$. $N^{*}$. In averaging $x_{\text {int }}^{\kappa, N^{*}}(z)$ over $z$, the region near the surface (within $1.5 \mathrm{~nm}$ of the outermost layer of solid atoms) was excluded because the fluid density in this region tends to be dominated by packing effects rather than interfacial physics.

Fig. $2 \mathrm{~b}$ and $3 \mathrm{~b}$ show $\bar{x}_{\text {int }}^{\kappa, N^{*}}$ vs. $N^{*}$ for the surfaces with $\varepsilon_{\mathrm{SW}}=1.94$ and $0.001 \mathrm{~kJ} \mathrm{~mol}^{-1}$, respectively. The results for all the surfaces considered are summarized in Table 1 , and highlight that in agreement with eqn (1), $h_{\mathrm{COM}}$ is $13-17 \%$ smaller than $h$.

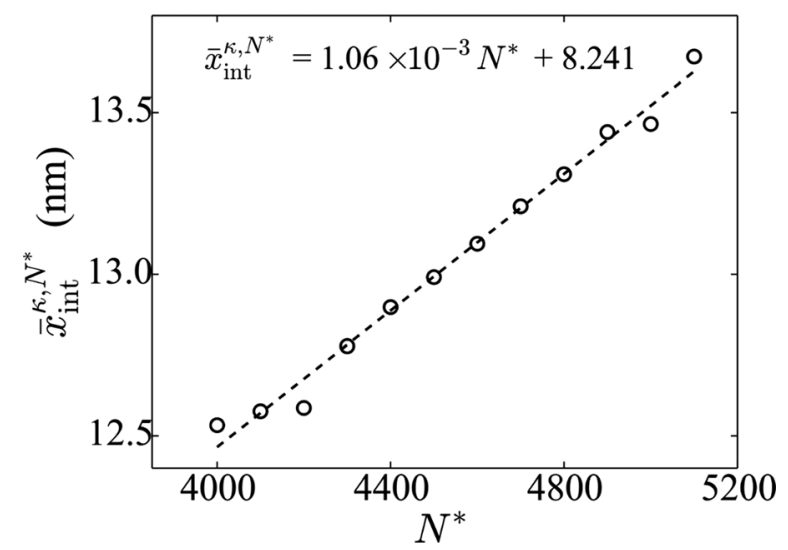

Fig. 2 (b) The variation of $\bar{x}_{\text {int }}^{\kappa, N^{*}}$ with $N^{*}$ (symbols) is shown for the LJ surface with $\varepsilon_{\mathrm{SW}}=1.94 \mathrm{~kJ} \mathrm{~mol}^{-1}$. The dashed line is a linear fit to the simulation data. Department of Chemical and Biomolecular Engineering, University of Pennsylvania, Philadelphia, PA 19104, USA. E-mail: amish.patel@seas.upenn.edu

$\dagger$ Electronic supplementary information (ESI) for this correction notice showing the derivation of eqn (1) is available alongside the original article, DOI: 10.1039/c8sm02317d 


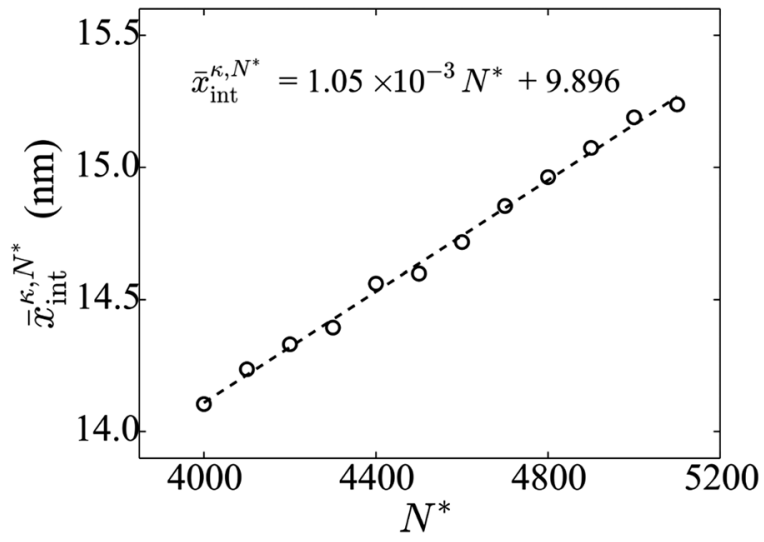

Fig. 3 (b) The variation of $\bar{x}_{\text {int }}^{\kappa, N^{*}}$ with $N^{*}$ (symbols) is shown for the LJ surface with $\varepsilon_{\mathrm{sw}}=0.001 \mathrm{~kJ} \mathrm{~mol}^{-1}$. The dashed line is a linear fit to the simulation data.

Table 1 For surfaces with different surface-water attractions, $\varepsilon_{\mathrm{SW}}$, the slope, $h_{\mathrm{int}}$, obtained by fitting $\bar{x}_{\text {int }}^{\kappa, N^{*}} v s$. $N^{*}$ to a straight line is shown, and is compared against $h_{\mathrm{COM}}$. The values of $h_{\mathrm{COM}} / h_{\text {int }}$ are roughly independent of $\varepsilon_{\mathrm{SW}}$, and in good agreement with eqn (1); for our simulation setup, $\alpha=0.23$, and $\lambda \approx 0.63$ (for the typical $\left\langle\tilde{N}_{v}\right\rangle_{\kappa, N^{*}} \approx 4500$ ), resulting in $\frac{h_{\mathrm{COM}}}{h} \approx 0.85$

\begin{tabular}{lll}
\hline$\varepsilon_{\mathrm{SW}}\left(\mathrm{kJ} \mathrm{mol}^{-1}\right)$ & $h_{\text {int }} \times 10^{3}(\mathrm{~nm})$ & $h_{\mathrm{COM}} / h_{\mathrm{int}}$ \\
\hline 0.001 & $1.05(2)$ & $0.87(3)$ \\
0.5 & $1.02(3)$ & $0.87(3)$ \\
1 & $1.05(2)$ & $0.85(2)$ \\
1.5 & $1.05(2)$ & $0.85(2)$ \\
1.94 & $1.06(3)$ & $0.83(3)$ \\
2.4 & $1.14(4)$ & $0.83(3)$
\end{tabular}

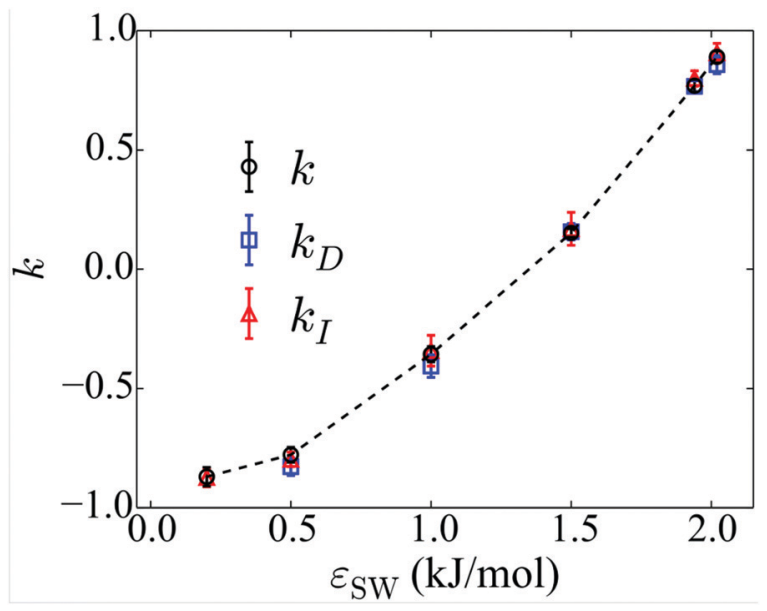

Fig. 4 (a) Wetting coefficients, $k$, estimated from SWIPES using $h_{\text {int. }}$. Due to a cancellation of errors, the estimates of $k$ agree well with those originally reported (as $k_{\mathrm{F}}$ ). Also shown for comparison are $k_{\mathrm{D}}$ and $k_{\mathrm{l}}$, which are computed as before.

Our use of $h_{\mathrm{COM}}$ thus led to $\gamma_{\mathrm{VL}}(\propto 1 / h)$ being overestimated $\left(\gamma_{\mathrm{VL}}^{\mathrm{COM}}=62(2) \mathrm{mJ} \mathrm{m}{ }^{-2}\right)$; using $h_{\text {int }}$ instead results in an estimate of $\gamma_{\mathrm{VL}}^{\text {int }}=56(2) \mathrm{mJ} \mathrm{m}^{-2}$, which is consistent with the values reported in the literature, once differences in the cutoff distances for the Lennard-Jones potential are accounted for. Note that our previous comparison to the literature did not account for such differences. Our use of $h_{\mathrm{COM}}$ also led to $k \gamma_{\mathrm{VL}}$ being overestimated by roughly $15 \%$; however, our use of $\gamma_{\mathrm{VL}}^{\mathrm{COM}}$ to compute the corresponding wetting coefficients, $k_{\mathrm{COM}}$, resulted in a fortuitous cancellation of errors, such that approximating $h$ by $h_{\mathrm{COM}}$ did not lead to substantive errors in $k$; see Fig. 4a. In particular, this error cancellation resulted from the fact that $h_{\mathrm{COM}} / h_{\mathrm{int}}$ depends primarily on the system setup geometry (eqn (1)), and is more or less independent of $\varepsilon_{\mathrm{SW}}$ (Table 1).

In conclusion, our use of $x_{\mathrm{COM}}$ as a proxy for interface location, $H$ : (i) led to an error of roughly 15\% in our estimate of $\gamma_{\mathrm{VL}}$; (ii) did not affect our estimates of $k$ (within error); and (iii) did not change the main conclusions of this work. The authors would like to acknowledge Sean M. Marks for his role in identifying and correcting the issue discussed in this Correction notice.

The Royal Society of Chemistry apologises for these errors and any consequent inconvenience to authors and readers. 